\title{
EEG Measures Index Neural and Cognitive Recovery from Sleep Deprivation
}

\author{
Bryce A. Mander, ${ }^{1,2,5}$ Kathryn J. Reid, ${ }^{2}$ Kelly G. Baron, ${ }^{2}$ Tjoson Tjoa, ${ }^{2}$ Todd B. Parrish, ${ }^{3}$ Ken A. Paller, ${ }^{1,4}$ \\ Darren R. Gitelman, ${ }^{1,2}$ and Phyllis C. Zee ${ }^{1,2}$ \\ ${ }^{1}$ Northwestern University Interdepartmental Neuroscience Program, and Departments of ${ }^{2}$ Neurology and ${ }^{3}$ Radiology, Feinberg School of Medicine, \\ Northwestern University, Chicago, Illinois 60611, ${ }^{4}$ Department of Psychology, Northwestern University, Evanston, Illinois 60208, and ${ }^{5}$ Department of \\ Psychology, University of California, Berkeley, Berkeley, California 94720
}

\begin{abstract}
Sleep deprivation impairs many cognitive abilities, but these impairments can be reversed after a certain quantity and quality of sleep. The ability to inhibit responding is particularly susceptible to disruption after prolonged wakefulness. How recovery sleep (RS) alters brain activity, leading to improved performance on a variety of cognitive tasks, remains unclear. This issue was examined in the current study using spectral analysis of electroencephalogram (EEG) data during sleep. These measures of sleep physiology were acquired after both normal sleep (NS) and RS, and were related to measures of inhibitory control and concurrent brain activity. Subjects were nine young adults who underwent functional magnetic resonance imaging twice, after $9 \mathrm{~h}$ of NS and after $10 \mathrm{~h}$ of RS that followed $38 \mathrm{~h}$ of being awake. A multiple regression model was used to examine differences between conditions in (1) EEG spectral power during sleep, (2) probability of successful inhibition in a go/no-go task, and (3) activation within a region of right prefrontal cortex during the task. Performance recovery, as indexed by reduced performance differences between conditions, was predicted by increased delta power and decreased sigma power in RS compared with NS. These EEG variables predicted most of the variance in inhibitory performance difference between conditions. Regressions also suggested that RS improved performance because of changes in brain function including prefrontal regions that resulted from delta rebound. We thus propose that slow waves, reflected in delta power during RS, act to restore brain function, thereby improving cognitive performance that entails response inhibition.
\end{abstract}

\section{Introduction}

Cognitive functioning declines without satisfactory sleep. Functions relying on the prefrontal cortex are among the most susceptible (Harrison et al., 2000; Thomas et al., 2000; Chuah et al., 2006; Wu et al., 2006). The nature of the neural dysfunction that produces these impairments remains unclear. These impairments are proposed to be tied to changes in the brain because of continuous wakefulness such as decreased metabolic resources available in the brain, or oxidative damage in neurons (Tononi and Cirelli, 2003; Schulze, 2004). Whatever the cause of these impairments, an examination of how the brain recovers from these effects may provide critical insight into the neural mechanisms underlying cognitive impairments resulting from sleep

\footnotetext{
Received Aug. 15, 2009; revised 0ct. 20, 2009; accepted Dec. 22, 2009.

This work was supported by Research Grant M01 RR-00048 from the National Center for Research Resources, Grant P01 AG11412 from the National Institute on Aging, Alzheimer's Disease Core Center Grant AG1385, and Grant F31 MH074291 from the National Institute of Mental Health, as well as support from the Northwestern University Cross-School Initiative. The present work was conducted at Northwestern University as part of Dr. Mander's dissertation. This was not an industry-supported study. We thank Dr. Lisa Wolfe and Dr. Prasanth Manthena, who conducted all the history and physicals for the study participants, and Bryan Carter, Nondas Leloudas, Renata Nikolayev, and the GCRC stafffor their assistance in running the protocol. We also thank the study participants for their time and commitment to research.

All of the authors have indicated no financial conflicts of interest.

Correspondence should be addressed to Bryce A. Mander, Department of Psychology, University of California, Berkeley, Tolman Hall, Suite 5305, University of California, Berkeley, Berkeley, CA 94720-1650. E-mail: bamander@ berkeley.edu.

DOI:10.1523/JNEUROSCI.4010-09.2010

Copyright $\odot 2010$ the authors $\quad 0270-6474 / 10 / 302686-08 \$ 15.00 / 0$
}

loss, recovery after sleep, and the normal benefits that sleep provides.

Sleep deprivation is followed by a rebound of sleep that is more intense (Kleitman, 1963; Carskadon and Dement, 1994). Characteristics of this increased intensity include (1) reduced responsiveness to the environment; (2) altered electroencephalographic properties (e.g., increased delta power and decreased sigma power, corresponding to increased slow-wave amplitude during slow-wave sleep and decreased sleep spindle activity, respectively); and (3) increased sleep time and efficiency (Patrick and Gilbert, 1896; Blake and Gerard, 1937; Kleitman, 1963; Kales et al., 1970; Borbély et al., 1981). Increased delta power during recovery sleep is most prominent over the frontal cortex, where improved function induced by recovery sleep may be most needed (Cajochen et al., 1999). With recovery sleep, performance improves (Patrick and Gilbert, 1896; Williams et al., 1959; Rosa et al., 1983; Bonnet, 1985; Cajochen et al., 1999; Gosselin et al., 2005), though one night may not be enough to fully recover prefrontal function (Wu et al., 2006). Taken together, these data suggest that slow-wave sleep may be critical for the recovery of prefrontal functions impaired by sleep loss. Data from mice support this idea in that increased delta power during recovery sleep seemed to specifically promote performance recovery in cognitive domains relying on prefrontal function (Bjorness et al., 2009). However, no measures of prefrontal activity were linked to these changes in performance and delta power. Further analyses of sleep physiology after prolonged wakefulness may 
A

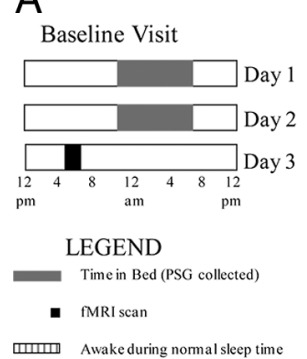

$\mathrm{B}$

Cue Stimulus End-Trial Next Trial Presentation Interval

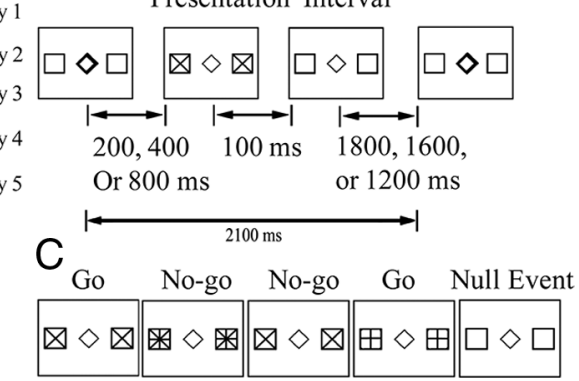

Figure 1. $\quad \boldsymbol{A}-\boldsymbol{C}$, Study protocol $(\boldsymbol{A})$ and schematic representation of the go/no-go task, showing a single trial $(\boldsymbol{B})$ and multiple trials $(\boldsymbol{C})$. In the study protocol $(\boldsymbol{A})$, subjects were studied on two separate occasions for a baseline visit and a sleep deprivation visit. Solid gray bars represent periods of polysomnographic (PSG) recordings of sleep, and the unfilled bar represents a period where participants remained awake when they would normally sleep. Solid black bars represent fMRI scans. On the go/no-go task (B), each trial consisted of fixation display followed by a nonspatial cue presented for 200,400 , or $800 \mathrm{~ms}$. After the cue, a stimulus was presented for $100 \mathrm{~ms}$ followed by an intertrial interval. An example of a series of five trials is presented ( $\boldsymbol{C}$. The correct response pattern is go/no-go/no-go/go, inhibiting responses to nontarget and lure events, respectively. The fifth trial represents the intermittent inclusion of null events, which last between 2.1 and $6.3 \mathrm{~s}$.

thus shed light on this hypothesized prefrontal role in recovery. Specifically, does the increased delta power associated with recovery sleep predict altered prefrontal activity, and does this alteration predict prefrontal-dependent performance recovery?

To this end, we compared delta and sigma power across $9 \mathrm{~h}$ of normal sleep (NS) to that observed across a night of $10 \mathrm{~h}$ of recovery sleep (RS) after 38 h of continuous wakefulness. In addition, we measured go/no-go task performance and concomitant prefrontal activation in each individual after NS and RS. The go/no-go task measures context-dependent responding that is dependent on the prefrontal cortex and is impaired by sleep deprivation (Garavan et al., 1999; Harrison et al., 2000; Chuah et al., 2006). Together, these data allow us to determine whether aspects of sleep physiology are associated with prefrontaldependent performance and neural recovery.

\section{Materials and Methods}

Subjects. Nine young adults $(26.0 \pm 1.2$ years, 4 female $)$ participated in the study. Participants were recruited through flyers and word of mouth. No participant had a habitual intake of caffeine greater than two cups of coffee per day or equivalent, and no greater than seven drinks of alcohol per week. All participants had no history of significant medical, neurological, or psychiatric illness. Participants reported being right-handed with a mean Edinburgh handedness score of $86.3 \pm 3.6$ on a scale of -100 (left handed) to +100 (right handed) (Oldfield, 1971). All research participants gave written informed consent, and this study was approved by the Institutional Review Board at Northwestern University.

Protocol. Study participants were admitted to the General Clinical Research Center (GCRC) on two separate occasions, completing a protocol for normal sleep (NS) on the baseline visit and a protocol for sleep deprivation (SD) and recovery sleep (RS) on the sleep deprivation visit, as shown in Figure $1 \mathrm{~A}$. Sleep was monitored for 1-2 weeks before each GCRC visit using sleep logs and wrist actigraphy (Mini Mitter) to ensure that participants had complied with their self-reported sleep habits. These habits had to fulfill the following criteria: spend between 7 and $9 \mathrm{~h}$ time in bed per night on average, bed time between 10 P.M. and midnight, and wake time between 5 A.M. and 9 A.M. During this prestudy period, participants were instructed to abstain from caffeine and alcohol intake, and participants were not provided with access to caffeine or alcohol while in the GCRC in any condition. The order of each visit was counterbalanced in a crossover design with participants acting as their own controls. On the first visit to the GCRC, every research participant underwent a full polysomnography (PSG) to screen for the presence of sleep disorders. Research participants awoke at the same time of day in all condi- tions and completed all scans at the same time of day. These scans were conducted in the afternoon to early evening (between 4 P.M. and 6 P.M.).

At the beginning of the baseline and sleep deprivation visits, each subject entered the GCRC two evenings before scanning. All participants were allowed $9 \mathrm{~h}$ time in bed to sleep on both nights during which sleep was recorded via PSG. After the first night of sleep recording, participants were allowed to leave during the day, and activity was monitored with wrist actigraphy. After the second PSG recording, participants remained in the GCRC until after scanning. In the NS condition, $10-12 \mathrm{~h}$ after awakening, each participant was scanned while performing a go/no-go task. In the SD condition, after the second PSG recording, participants remained awake within the GCRC for $38 \mathrm{~h}$ and were constantly monitored by research staff to make sure the participant did not fall asleep. During their time in the GCRC, subjects were allowed to watch television, read, and interact and play games with the research staff. In the RS condition, participants were allowed $10 \mathrm{~h}$ time in bed in the GCRC to recover from sleep deprivation. In the afternoon, 10-12 $\mathrm{h}$ after awakening, each participant was scanned while performing the same go/no-go task. Participants were constantly monitored by research staff at all times in the GCRC to make sure they did not fall asleep.

Polysomnogram recording and analysis. Sleep was assessed using PSG, including four scalp electrodes for monitoring EEG at central $(\mathrm{C} 3, \mathrm{C} 4)$ and occipital $(\mathrm{O} 1, \mathrm{O} 2)$ locations, with a reference electrode on the ear on the opposite side of the head (A1,A2). In addition, electrooculogram, chin electromyogram (EMG), and electrocardiogram were obtained on all nights. Nasal/oral airflow, abdominal and chest respiration, pulse oximetry, and leg EMG were additionally monitored during the first night to screen for the presence of sleep disorders. Signals were amplified and sampled at $200 \mathrm{~Hz}$ (Neurofax EEG-1100, Nihon-Kohden), with a 70 $\mathrm{Hz}$ low-pass filter and a time constant of $0.3 \mathrm{~s}(0.6 \mathrm{~Hz})$. Recordings were scored according to the criteria of Rechtschaffen and Kales (1968).

Each study participant had five nights of sleep recording. Two recordings occurred before each experimental visit (baseline visit night 1 and 2 and sleep deprivation visit night 1 and 2) (Fig. 1A). These were all baseline nights with $9 \mathrm{~h}$ of time in bed, though night 1 for each visit was considered a habituation night. For all analyses of sleep variables, baseline visit night 2 and sleep deprivation visit night 2 were averaged and used as normal sleep (NS) condition data. The night after sleep deprivation was considered the recovery sleep (RS) condition, and consisted of $10 \mathrm{~h}$ of time in bed. Baseline and recovery characteristics for total recording time (TRT), total sleep time (TST), sleep latency, sleep efficiency, percentage of sleep period spent in wake, stage I, stage, II, slow-wave sleep, and rapid eye movement (REM) sleep were analyzed. Total recording time was defined as the period between lights off and lights on. Sleep onset and morning awakening were defined as, respectively, the times of the first and last $30 \mathrm{~s}$ intervals scored as non-rapid eye movement (NREM) or REM sleep. Total sleep time (TST) was defined as the time interval separating sleep onset from morning awakening minus the amount of time spent awake during the night. The sleep latency was defined as the time interval separating lights off from stage 2 onset. Sleep efficiency was calculated as the bedtime period minus the total duration of awakenings, expressed as the percentage of the bedtime period. Wake after sleep onset was defined as the period of time spent awake after sleep onset and before lights on. Because the time in bed differed between conditions, percentage of stages I, II, III, IV, and REM sleep, and wake were analyzed. Sleep stages III and IV were combined as a measure of slow-wave sleep. A paired $t$ test was used to determine sleep condition (NS, RS) effects on sleep characteristics. All sleep data were analyzed using SPSS version 16.0 (SPSS). 
Spectral analysis of PSG data. EEG data were analyzed using spectral analysis. This analysis was conducted on central and occipital EEG leads using an electrophysiological recording analyzer software package (PRANA, Phitools). This software contains an automated artifactdetection algorithm that removes electrode detachment, overflow, flatline, power-line, ocular, muscular, and movement artifacts, which may confound spectral analysis. Following this automated procedure, individual records were visually inspected for verification of the procedure and removal of additional artifacts. After artifact removal, a fast Fourier transform was applied to the EEG signal at 4 s intervals with $50 \%$ overlap, giving a frequency resolution of $0.25 \mathrm{~Hz}$. A Hanning window was used, minimizing the influence of the ends of each epoch on spectral analysis of frequencies. Power spectra from corresponding epochs were averaged into $30 \mathrm{~s}$ measurements to align these measurements with sleep staging. Spectral analysis of absolute power was conducted across four frequency ranges: delta $(0.5-4.5 \mathrm{~Hz})$, theta $(4.5-8.5 \mathrm{~Hz})$, alpha $(8.5-12.5 \mathrm{~Hz})$, and sigma $(12.5-15.5 \mathrm{~Hz})$. In the current report, data are presented for the central EEG lead C3, though data were examined at C4 and determined to be highly correlated with C3 data.

As with sleep-staging analysis, baseline visit night 2 and sleep deprivation visit night 2 were averaged and used as NS condition data. Overnight averages of delta and $\sigma$ power were calculated during NREM sleep periods across the first $9 \mathrm{~h}$ in both NS and RS conditions. This allowed for an equal time in bed comparison between baseline and recovery sleep nights. A paired $t$ test was used to determine sleep condition effects (NS, RS). Percentage differences in delta, theta, alpha, and sigma power between NS and RS conditions were calculated and used as independent regressors within multiple regression models comparing the effects of spectral power change on inhibitory performance differences and concomitant prefrontal activation differences.

Go/no-go task. The go/no-go task was designed to examine inhibition of prepotent responses and is a variant of one described by Garavan et al. (1999). Figure 1, $B$ and $C$, illustrates trial organization and timing, and details for each task run are included in the supporting information section. Participants were instructed to fixate centrally on a diamond throughout the experiment. On each trial, a stimulus appeared in peripheral boxes located $7^{\circ}$ to the left and right of fixation, always the same stimulus in both boxes. Participants were instructed to press a "go" response key in an alternating manner to " $X$ " or " + " stimuli (targets), and to inhibit responding "no-go" when the same target symbol repeated (lures: " $X$ " following " $X$ " or " + " following " + ") or whether any other symbol appeared (nontargets). Trials included a random sequence of targets (53\%), lures (18\%), and nontargets (29\%). Each stimulus was preceded by a cue (darkening of the fixation diamond) with temporal expectancy minimized by using a stimulus onset asynchrony that varied randomly among 200,400 , and $800 \mathrm{~ms}$. The intertrial interval varied as a function of these delays to maintain a trial length of $2.1 \mathrm{~s}$. Each participant completed two functional magnetic resonance imaging (fMRI) runs of the task during the normal sleep (NS), sleep deprivation (SD), and recovery sleep (RS) conditions.

Each experimental run contained 152 trials ( 81 targets, 44 nontargets, and 27 lures). Fifty null events were distributed throughout the run to allow deconvolution of the hemodynamic response function. These events consisted of a fixation display for 2.1-6.3 s. To train participants, the task was practiced before sleep on the second night of both visits. Practice versions of the go/no-go task had a higher proportion of targets to lures, such that expectations were biased toward expecting targets, thus enhancing response prepotency during scanning (100 targets, 42 nontargets, and 10 lures).

Behavioral analysis. Behavioral data collected within the scanner for NS, SD, and RS conditions were analyzed for this report. Percentage of no-go trials (lures, nontargets) correctly inhibited and percentage of go trials (targets) correctly responded to were calculated for each participant in each condition. A one-way, repeated-measures ANOVA was used to compare each percentage of correct inhibitions and responses across conditions (NS, SD, RS). To determine individual differences in inhibitory performance recovery, two methods were used: (1) percentage of correct inhibitions was compared between NS and RS conditions; and (2) percentage of correct inhibitions was compared between SD and RS con- ditions. For method 1, to determine whether performance in the RS condition was related to the performance deficit directly preceding it (in the SD condition), performance in the SD condition was correlated with performance in the RS condition, and the difference between performance in the NS and RS conditions was compared with the difference between performance in the NS and SD conditions. To determine whether performances in the RS and SD conditions were more related to each other than to the NS condition, a multiple regression model was used with RS and NS data predicting SD data. This would argue that analyses comparing performance difference (NS vs RS) with EEG and brain activity differences reflect neural correlates of the recovery process from SD and not just differences across $2 \mathrm{~d}$. Performance differences between NS and RS conditions and SD and RS conditions were compared to determine whether these measures of recovery were equivalent or distinct. All behavioral data were analyzed using SPSS version 16.0 (SPSS).

MRI scanning. Subjects were imaged using a Siemens Trio 3.0-T scanner equipped with a transmit and receive head coil. Anatomical scans were acquired in axial planes parallel to a plane through the anterior and posterior commissure. $\mathrm{T}_{1}$-weighted anatomical images were obtained using a 3D MPRAGE protocol with the following parameters: repetition time (TR), $2100 \mathrm{~ms}$; echo time (TE), $4.38 \mathrm{~ms}$; flip angle, $8^{\circ}$; field of view (FOV), $220 \mathrm{~mm}$; matrix, $256 \times 256$; slice thickness, $1.0 \mathrm{~mm}$; and 160 slices. Next, functional scans were acquired at the same orientation $(3 \times$ $3 \times 3 \mathrm{~mm}$ resolution) using a susceptibility-weighted, single-shot EPI method to image the regional distribution of the blood oxygenation level-dependent signal (TR/TE, 2100/30 ms; flip angle, 90 FOV, 220 $\mathrm{mm}$; matrix, $64 \times 64 ; 34$ contiguous $3 \mathrm{~mm}$ slices). In all functional runs, the magnetic resonance signal was allowed to reach equilibrium over the six initial scans, which were excluded from analysis.

In the scanner, participants viewed images that were projected onto a nonmagnetic screen located $\sim 65 \mathrm{~cm}$ from their eyes. Head movement was reduced by using a vacuum pillow (VacFix) and a cloth collar (Scott Specialties). Subjects responded using a fiberoptically linked button.

fMRI analysis. Functional data were analyzed using SPM5 (Wellcome Department of Imaging Neuroscience) running under a Matlab environment (Mathworks). Functional images were slice timing corrected, realigned, and then coregistered to the anatomic $\mathrm{T}_{1}$ volume. The $\mathrm{T}_{1}$ volume was then normalized to the Montreal Neurolgical Institute (MNI)-305 template supplied with SPM5. The template approximates the space described in the atlas of Talairach and Tournoux (1988).

At the individual subject level, neural responses to correct inhibitions (no-go), errors of commission (error), correct responses (go), and errors of omission (lapse) were modeled independently. Affine movement covariates were also included in the design matrix to model residual movement-related effects. Within each scanning session, subjects completed two versions of the task and underwent scanning twice. A covariate was included to control for effects of the task scanning session. NS and RS runs were modeled as separate sessions. The fMRI design matrix did not include a global covariate, as it can bias the parameter estimates (Aguirre et al., 1998). Instead, a voxel-level linear model of the global signal, which has been shown not to introduce bias, was used to remove the global effects (Macey et al., 2004).

Group activations were assessed by a second-level random-effects analysis, using a full factorial model with sleep condition (normal sleep, recovery sleep) and response type (no-go, error, go) as within-subject factors. Activity during go events were subtracted from no-go events to examine activity primarily related to inhibitory control by attempting to discount effects of attention, maintenance of information within working memory, and motor planning. We have used a similar contrast previously (Booth et al., 2003), and this contrast has been used by others on comparable tasks (Menon et al., 2001).

In the NS condition, whole-brain activation associated with the nogo/go contrast was examined. A cluster within the right prefrontal cortex was identified for a region of interest (ROI) analysis using the MarsBaR toolbox (Brett et al., 2002) (Fig. 2). This region was chosen because activations that overlap with these coordinates are reported in a large number of studies of response inhibition (Garavan et al., 1999; Konishi et al., 1999; Menon et al., 2001; Watanabe et al., 2002; Horn et al., 2003; 


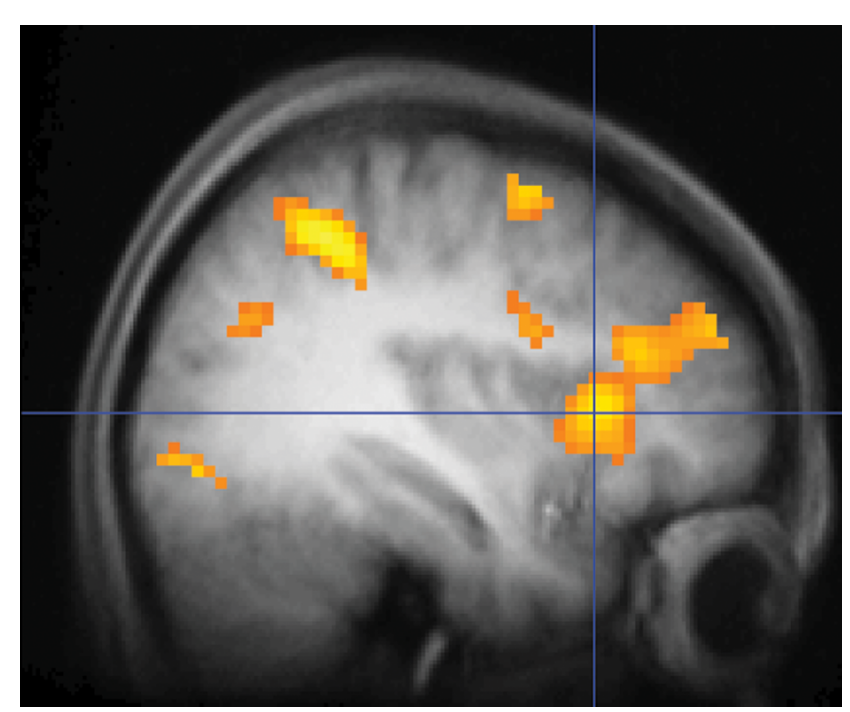

Figure 2. The cluster marked by the blue cross was extracted to examine right prefrontal activity after NS and RS (no-go/go contrast). The blue cross represents the coordinate of the cluster maxima $(x=36 ; y=21 ; z=6 ; 349$ voxels).

Bellgrove et al., 2004; Hester et al., 2004), the prefrontal hypothesis of sleep loss posits that the prefrontal cortex is most sensitive to sleep loss (Harrison et al., 2000; Thomas et al., 2000), and recent data suggest that the prefrontal cortex may not be fully recovered after one night of sleep (Wu et al., 2006). Our goal was to explore how task-related prefrontal activation difference between conditions statistically mediated how the differences in sleep parameters related to the inhibitory performance differences. Mean contrast estimates within the following cluster were extracted with the following coordinates as its maxima $(x=36 ; y=21$; $z=6 ; 349$ voxels).

To examine the relationship between activation change across conditions, inhibitory performance change across conditions, and spectral power density change in the delta and sigma bands across conditions, activity change within the prefrontal ROI from NS to RS conditions was included as a variable in two multiple regression models. In one model, percentage delta and sigma change were regressed against activation change to determine the effects of recovery sleep physiology on next-day prefrontal functioning. In a second model, right prefrontal activation change was included as an independent variable along with the percentage of delta and sigma change to predict inhibitory performance change. To determine whether the effects of delta and sigma power changes on inhibitory performance recovery were mediated by their effects on right prefrontal activity, Sobel tests of mediation were completed (MacKinnon et al., 1995). This Sobel test determines statistically how much the influence of independent variable $X$ on dependent variable $Y$ is accounted for by mediator $M$; i.e., is the value of the direct path coefficient between $X$ and $Y$ reduced by the inclusion of $M$ ? Reduction to 0 is generally interpreted as mediation, partial reduction is interpreted as partial mediation, and nonsignificant reduction is interpreted as no evidence for mediation. The same analyses were conducted using performance and right prefrontal activation change from SD to RS conditions. Analyses were completed using SPSS version 16.0 (SPSS). It is possible, particularly in low $n$ studies, that the strengths of these relationships were influenced by outliers. To address this concern, iteratively reweighted least squares (IRLS) was used for robust regression analysis (Holland and Welsch, 1977). This analysis was conducted in JMP version 8.0 (SAS Institute).

\section{Results}

\section{PSG data}

Many differences in sleep physiology were apparent between the NS and RS conditions, as shown in Table 1. The total amount of RS was an average of 111 min more than NS. Sleep latency, wake after sleep onset, and percentage of stage 1 and 2 sleep were re-
Table 1. Sleep variables

\begin{tabular}{|c|c|c|c|c|}
\hline \multirow[b]{2}{*}{ PSG variable } & \multicolumn{2}{|l|}{ Sleep condition } & \multicolumn{2}{|c|}{ Test statistic } \\
\hline & Normal sleep & Recovery sleep & $t_{(8)}$ & $p$ \\
\hline TRT (h) & $9.05(0.02)$ & $10.08(0.03)$ & -25.222 & $<0.001$ \\
\hline TST (h) & $7.89(0.15)$ & $9.74(0.05)$ & -13.676 & $<0.001$ \\
\hline Sleep latency (min) & $28.97(3.80)$ & $5.28(1.24)$ & 6.094 & $<0.001$ \\
\hline Sleep efficiency (\%) & $92.95(1.17)$ & $97.68(0.39)$ & -5.416 & 0.001 \\
\hline WASO (\%) & $5.54(1.06)$ & $1.07(0.33)$ & 5.171 & 0.001 \\
\hline Stage 1 sleep (\%) & $0.72(0.11)$ & $0.17(0.07)$ & 3.896 & 0.005 \\
\hline Stage 2 sleep (\%) & $54.59(1.30)$ & $50.37(2.19)$ & 3.156 & 0.013 \\
\hline Slow-wave sleep (\%) & $11.90(1.16)$ & $19.50(1.76)$ & -8.756 & $<0.001$ \\
\hline REM sleep (\%) & $25.74(1.21)$ & $27.69(2.22)$ & -1.063 & 0.319 \\
\hline Mean delta power $\left(\mu V^{2}\right)$ & $1245.6(195.2)$ & $1824.5(278.5)$ & -3.826 & 0.005 \\
\hline Mean theta power $\left(\mu V^{2}\right)$ & $83.5(13.9)$ & $109.5(21.9)$ & 2.871 & 0.021 \\
\hline Mean alpha power $\left(\mu V^{2}\right)$ & $34.6(7.2)$ & $43.9(10.2)$ & 2.133 & 0.066 \\
\hline Mean sigma power $\left(\mu V^{2}\right)$ & $25.1(5.0)$ & $25.7(4.7)$ & -0.668 & 0.523 \\
\hline
\end{tabular}

Values are expressed as the mean (SEM). TRT, Total recoding time; Sleep latency, time to first stage 2 epoch; WASO, wake after sleep onset.

Table 2. Go/no-go performance

\begin{tabular}{lccccccc}
\hline & \multicolumn{2}{l}{ Sleep condition } & & \multicolumn{2}{c}{ Test statistic } \\
\cline { 2 - 3 } Performance variable & NS & SD & RS & & $F_{(2,16)}$ & $p$ \\
\hline Correct inhibitions (\%) & $92.6(1.9)$ & $84.4(3.3)$ & $89.6(3.2)$ & & 7.81 & 0.004 \\
Correct responses (\%) & $97.5(1.6)$ & $88.1(3.4)$ & $94.4(2.2)$ & & 3.95 & 0.040 \\
MRT correct responses (ms) & $322.2(22.2)$ & $331.4(17.0)$ & $320.9(19.5)$ & & 0.53 & 0.596 \\
SD correct responses (ms) & $77.3(7.3)$ & $99.2(8.7)$ & $85.2(8.2)$ & & 7.36 & 0.005 \\
\hline
\end{tabular}

Values are expressed as the mean (SEM). MRT, Mean reaction time.

duced during NS compared with RS. Sleep efficiency and percentage of slow-wave sleep were greater in RS compared with NS. Delta power was $\sim 50 \%$ greater in RS than NS, theta and alpha power were $30 \%$ greater in RS than NS, whereas sigma power did not differ significantly. Delta power during NREM sleep is thought to primarily reflect the power of slow waves, whereas sigma power primarily reflects the power of sleep spindles (Borbély et al., 1981). Some data suggest that during recovery sleep, delta power increases and suppresses sigma power (Dijk et al., 1993). To investigate a possible delta suppression of sigma, a correlational analysis was conducted. No apparent relationship was detected between the change in delta and sigma power between NS and RS conditions $(F=1.370, r=-0.405, p=0.280)$. Delta and sigma power were highly correlated between the two baseline nights $\left(r^{2}=0.911, p=0.0002\right.$ for delta power and $r^{2}=$ 0.987, $p<0.0001$ for sigma power), and using spectral data from sleep deprivation visit night 2 as the NS data produced similar results as the average between baseline visit night 2 and sleep deprivation visit night 2 (available at www.jneurosci.org as supplemental material). These data suggest the effects in the current study were not driven by a noisier power spectrum during the RS condition.

\section{Behavioral data}

Significant condition effects were detected for the percentage of correct inhibitions, the percentage of correct responses, and the SD of reaction time for correct responses (Table 2). Bonferroni post hoc testing revealed all these effects were indicative of sleep deprivation (SD), resulting in fewer correctly inhibited trials, fewer correct response trials, and increased SD of reaction time on correct response trials. No other comparisons were significant. One night of recovery sleep after 38 h of continuous wakefulness thus resulted in recovered go/no-go task performance on a group level. However, there was high interindividual variability in inhibitory performance difference between NS and RS conditions varied across individuals, ranging from a correct inhibition 
percentage that was $4.9 \%$ worse in NS than in RS to a correct inhibition percentage that was $15.5 \%$ better in NS than RS, with a mean NS-RS difference of $3.0 \pm 1.9 \%$. Individual differences in the recovery condition were correlated with individual differences in the SD condition $(r=0.87$, $p=0.002$ ), and the difference between NS and RS was correlated with the difference between NS and SD $(r=0.78, p=0.014)$. Finally, a multiple regression model including NS and RS performance data as predictors of SD performance explained a large portion of the SD performance variance $\left(r^{2}=0.790, p=0.0093\right)$, but only RS performance remained a significant predictor $(p=0.3638$ for NS performance, $p=0.0135$ for RS performance), suggesting that in terms of inhibitory performance, performance after RS is particularly related to the SD impairment that directly preceded it. This argues that analyses comparing performance difference (NS vs RS) with EEG and brain activity differences reflect neural correlates of the recovery process from SD and not just differences across $2 \mathrm{~d}$. Finally, performance difference between NS and RS was compared with performance difference between SD and RS conditions. No significant relationship was detected $(r=0.06, p=0.872)$, suggesting that these two measures of performance recovery are unrelated and may track with distinct EEG and fMRI signals.

\section{Functional MRI data}

Similar to the behavioral data, no significant differences in brain activity were detected at a group level as a function of condition. Activity within a right prefrontal volume of interest that was greater for no-go than go events (no-go/go contrast) was examined (Fig. 2). Mean parameter estimates within this cluster, for the no-go/go contrast after NS, were extracted using the MarsBaR toolbox within SPM5 (Brett et al., 2002) and regressed against the percentage of no-go trials successfully inhibited after NS. A significant relationship was detected $(F=5.461, r=-0.662, p=$ $0.052)$. Responses within the right prefrontal cortex volume of interest were similar in NS and RS conditions $\left(t_{(8)}=0.817, p=\right.$ 0.438 ; for NS, $0.85 \pm 0.12$; for RS, $0.72 \pm 0.19$ ). However, the difference in activation from NS to RS was significantly associated with the difference in inhibitory performance across individuals $(F=41.998, r=-0.926, p<0.001)$ (Fig. $3 E)$. The same relationship was not detected when comparing change in activation from SD to RS conditions with inhibitory performance change from SD to RS conditions $(r<0.01, p=0.994)$. When inhibitory performance change from SD to RS conditions was included as a regressor in a whole-brain model, a significant negative relationship was detected within a small cluster within the left visual cortex $(x=-15 ; y=-63 ; z=-15 ; z=4.35 ; 33$ voxels). However, right prefrontal activation change from SD to RS was correlated with performance change from NS to RS ( $r=$ $0.775, p=0.0142$ ). Further, changes in activation from NS to RS and from SD to RS were more related to the performance change from NS to RS than from SD to RS. In models including NS - RS performance change and RS - SD performance change as predictors of prefrontal activity change from NS to RS and from SD to RS, NS - RS performance predicted activation change for both NS to RS and SD to RS models; whereas, RS - SD performance change did not predict either (for right prefrontal activation
B
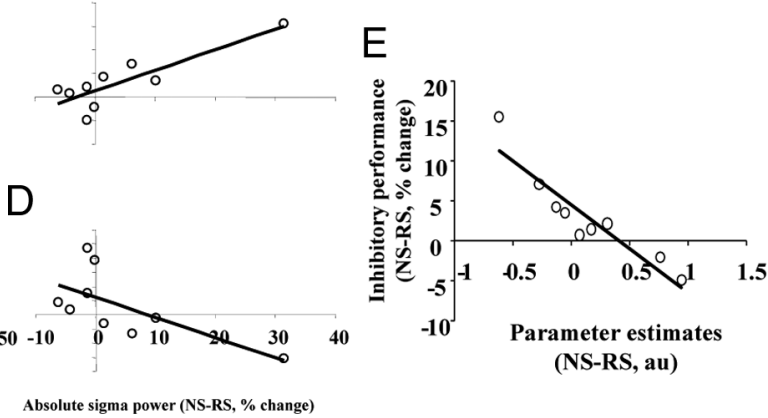

(NS-RS, au)

\section{Sleep variables as predictor variables}

The degree to which delta and sigma EEG power differed between NS and RS was found to systematically relate to task performance difference across conditions. A larger delta power difference between NS and RS predicted a smaller performance difference between NS and RS $\left(B=-0.117, r^{2}=0.495, p=0.034\right)$ (Fig. $3 A, C)$. Reciprocally, a larger sigma power difference between NS and RS predicted a larger performance difference between NS and RS $\left(B=0.434, r^{2}=0.727, p=0.003\right)$ (Fig. $\left.3 B, D\right)$. Together, delta and sigma power change predicted a large portion of the inhibitory performance variance $\left(r^{2}=0.881, p=0.0017\right)$. When prefrontal activation was included in the regression model, delta power was not significant ( $p=0.695$ ), and sigma and prefrontal effects remained significant ( $p=0.0246$ and 0.023 , respectively). Sobel tests of mediation (MacKinnon et al., 1995) determined that prefrontal activation-mediated effects of delta and partially mediated effects of $\sigma$ on inhibitory performance ( $p=0.0097$ and 0.03 , respectively). These data suggest that delta power may exert its effects on inhibitory performance via its effects on right prefrontal cortex, while sigma power may influence inhibitory performance via additional neural mechanisms. This effect was not explained by raw absolute power differences between subjects during NS ( $r=0.216, p=0.5769)$ or RS $(r=0.135, p=0.7284)$. When absolute delta power for NS and RS were included in the model, percentage delta change remained a trend $(p=0.0600$ and 0.0542 , respectively); whereas, absolute delta remained nonsignificant ( $p=0.9196$ and 0.8998 , respectively). However, it is possible that the strength of these relationships was influenced by outliers. To address this concern, iteratively reweighted least squares (IRLS) was used for robust regression analysis (Holland and Welsch, 1977). Robust regression analysis demonstrated that delta power difference was predictive of performance difference $\left(r^{2}=0.637, p=0.0099\right)$, whereas sigma power difference showed a trend toward predicting performance difference $\left(r^{2}=0.382, p=0.076\right)$.

To explore the specificity of these effects, we additionally examined the influence of total sleep time (TST) and alpha and theta power differences. TST did not predict performance recovery $(r=0.512, p=0.159)$, and when TST was included in the 
Table 3. Sigma power (RS-NS) versus activation (NS-RS; no-go vs go events)

\begin{tabular}{|c|c|c|c|c|c|c|}
\hline \multirow{2}{*}{$\begin{array}{l}\text { Age, brain region, and } \\
\text { contrast }\end{array}$} & \multirow{2}{*}{$\begin{array}{l}r \text { direction } \\
(+,-)\end{array}$} & \multicolumn{3}{|c|}{ MNI coordinates } & \multirow[b]{2}{*}{ z score } & \multirow[b]{2}{*}{ Voxel \# } \\
\hline & & $x$ & $y$ & $z$ & & \\
\hline Right superior frontal sulcus & + & 27 & 42 & 36 & 4.51 & 78 \\
\hline Left superior frontal sulcus & - & -36 & 3 & 63 & 4.45 & 27 \\
\hline Left superior parietal/precuneus & - & -15 & -75 & 54 & 3.94 & 29 \\
\hline
\end{tabular}

\#, Number. tween NS and RS conditions. Sigma power increases were associated with right superior prefrontal activation decreases and left superior prefrontal and parietal activation increases (Table 3, Fig. $4 A-C)$.

\section{Discussion}

These data demonstrate that recovery sleep alters next-day prefrontal activation, which in turn contributes to successful inhibitory performance recovery. When the go/ no-go task was performed after $10 \mathrm{~h}$ of RS, performance nearly reached normal levels, as estimated by comparison to performance in the NS condition. Thus, the performance difference between RS and NS conditions was taken as a behavioral index of the effectiveness of RS. In support of this, RS and SD performance were more related to each other than to NS performance. This suggests the RS condition represents a state where cognitive recovery from sleep loss is not complete. Furthermore, the effectiveness of RS as assessed in this manner was related to EEG characteristics observed during RS: (1) effectiveness was stronger with greater delta and theta in RS compared with NS; and (2) RS effectiveness was stronger with less sigma in RS compared with NS. Given the collinearity of delta and theta power, it remains difficult to distinguish whether delta and theta rhythms contribute to recovery independently or whether theta shows a relationship merely because of its model $\left(r^{2}=0.882, p=0.0093\right)$ with delta and sigma, it was not significant $(p=0.8183)$, but sigma remained significant $(p=$ $0.0131)$ and delta remained a trend (0.0650). Alpha power difference did not predict performance recovery $(r=0.093, p=$ $0.8124)$, and when alpha power difference was included in the model $\left(r^{2}=0.895, p=0.0070\right)$ with delta and sigma, it was not significant $(p=0.8183)$, but sigma and delta remained significant ( $p=0.0078$ and 0.0367 , respectively). The relationship between theta power difference and inhibitory performance difference showed a trend toward significance $(r=0.653, p=$ $0.0567)$. However, theta power change was highly correlated with delta power change $(r=0.873, p=0.0021)$. The combination of delta and theta power produced similar results as delta power alone (available at www.jneurosci.org as supplemental material), suggesting that spectral power change in the delta and theta frequency ranges predicts inhibitory performance recovery.

The relationship between delta and sigma power change and SD to RS performance change was not detected $(r=0.04, p=0.609$ and $r=0.03, p=0.641$, respectively), nor were any relationships between change in sleep and performance change, e.g., change in total sleep time, percentage change in slow-wave sleep, alpha and theta power change. Change in Delta power from NS to RS conditions predicted change in right prefrontal activation from $\mathrm{SD}$ to RS conditions $(r=0.779, p=0.0134)$, and change in sigma power did not $(r=0.47, p=0.2013)$. Change in right prefrontal activation from SD to RS conditions did not act as a mediator between delta and sigma power and inhibitory performance change from NS to RS or SD to RS.

To explore what additional neural mechanisms sigma may influence, percentage increase in sigma power was regressed against whole brain activation change (no-go/go contrast) be- correlation with delta. Thus, increasing the prevalence of slow waves (and perhaps NREM theta rhythms) and decreasing the prevalence of sleep spindles appears to restore the ability to inhibit responses in the go/no-go task. At a group level, inhibitory performance was recovered after one night of RS, and this was associated with a $\sim 50 \%$ increase in delta power. This effect was independent of absolute power after NS and RS, suggesting change in power between conditions predicts performance recovery, and not individual differences in absolute power.

The fMRI results provided additional insight into the neurophysiology and functional neuroanatomy of recovery sleep. Regression results showed that the effect of increased delta power on inhibitory performance was mediated by its effect on right prefrontal activation. Although prefrontal cortex may also be involved in mediating effects of sigma power changes, results suggested further mediation through other neural sources such as superior prefrontal and parietal cortex. Other frontal, parietal, and occipitotemporal regions were recruited by this task after NS (supplemental Table S1, available at www.jneurosci.org as supplemental material), but no activation in any of these regions mediated the relationship between sleep and performance recovery. This argues that delta rebound may restore inhibitory performance through action on relevant prefrontal systems. Given the relatively low power in the current study to detect relationships attributable to a small sample size, these effects may not be exclusive to the prefrontal cortex but may simply include the prefrontal cortex. Together, these data suggest that slow waves act to restore or preserve next-day brain function in areas including the prefrontal cortex, whereas sleep spindles may alter brain 
function in a way that is not conducive to inhibitory performance recovery.

The same relationships were not detected using changes from $\mathrm{SD}$ to RS as an index of performance recovery. These analyses suggest that the correlations we observed with NS to RS changes were specific to this method of measuring performance recovery. No relationships were observed with performance change from SD to RS and sleep, suggesting that change in sleep after sleep deprivation is more predictive of how close one is to baseline in terms of performance instead of how much one recovers in an absolute sense. How slow waves during slow-wave sleep lead to the restoration of function remains a mystery. Recent evidence suggests the homeostatic regulation of slow waves may be controlled by astrocytic influences on adenosine type 1 receptors (Halassa et al., 2009), and that this relationship has consequences for recovery of working memory performance in mice (Bjorness et al., 2009). Slow waves have been proposed to be important for processes such as dissipation of the homeostatic drive, neurometabolic regulation, memory, and cellular restitution, each of which may contribute to the process of performance recovery (Tononi and Cirelli, 2003; Schulze, 2004).

In the current study, delta power was measured from central electrodes as frontal electrodes were not applied. Though this is a limitation of the current study, in a separate set of individuals, we observed that delta power at the C3 derivation correlated very highly with delta power detected at the F3 and Fz derivations (available at www.jneurosci.org as supplemental material). Thus, delta power at the $\mathrm{C} 3$ derivation appears to be a good marker for delta power at frontal derivations. Future studies will need to examine whether localized changes in slow-wave activity predicts recovery of specific brain functions and related behaviors.

Whereas slow waves during slow-wave sleep are primarily generated within prefrontal cortex (Steriade et al., 1993; Massimini et al., 2004), sleep spindles are generated in the reticular nucleus of the thalamus and sculpt widespread corticothalamic activity (Steriade et al., 1987). Late-night stage 2 sleep, where sleep spindle amplitude is at its highest, has been associated with improved motor skills (Walker et al., 2002). This improvement has been associated with a functional change in task-related activation in sensory-motor brain regions (Walker et al., 2005), suggesting a transition toward a more automated response strategy. The task in the current study is a fairly simple visuomotor task with a high degree of built-up response prepotency. Those individuals that increased sigma power to a greater degree may have developed a more automated response strategy. This automated response strategy may have been less optimal for a task that requires the ability to inhibit a prepotent response. Thus, the degree of inhibitory performance recovery may depend on multiple physiological changes, which may rely on distinct physiologic systems.

Slow waves and sleep spindles are known to reciprocally inhibit each other (Dijk et al., 1993). This reciprocal relationship suggests that the interaction of delta and sigma power may explain performance recovery more fully. In other words, increased sigma power may only relate to performance recovery and concomitant activation change because of its relationship to delta power. In the current study, differences in delta and sigma power from NS to RS were not correlated across subjects, arguing against this explanation. To more fully examine this question of delta and sigma interaction, an interaction term was included in the multiple regression model (\% delta change $\times \%$ sigma change centered on the group mean), but was not found to be significant. It must be noted that the current study sample size is small, and delta power and sigma power explained a large portion of the variance in performance. Thus, the current study is not powered to examine whether spindle power is associated with inhibitory performance across conditions merely through its association with slow-wave intensity or independently of slow-wave intensity. However, if this sigma-performance relationship was due entirely to the suppression of spindles by slow waves, then we would expect equal mediation of prefrontal activity on the effects of both sigma and delta power. We did not see this, and instead show that sigma power remains a significant predictor when prefrontal activity is included into the regression model. This suggests that spindle action is at least partially independent of the influence of delta on brain function.

Sleep is a heterogeneous physiological process with multiple stereotypic wave forms associated with distinct EEG spectral bands within separate sleep stages. It is likely that these different waveforms may be important for the recovery of different functions. The current study is limited to a small sample size, tests only one cognitive domain, and examines brain activity in a targeted prefrontal region, and thus cannot answer this question. To better elucidate the relationships between sleep, cognitive recovery, and brain function, future studies with larger sample sizes that test performance over multiple cognitive domains will be necessary.

Another possibility is that sigma differences between NS and RS are not characteristic of differences in sleep spindle power per se, but rather are attributable to general differences in background sigma power (De Gennaro and Ferrara, 2003). In this case, increased sigma may represent a marker of sleep that was less restorative than sleep with lower general sigma power. Further, robust regression analysis revealed that the relationship between sigma power change and performance was influenced by outliers. When correcting for outliers, there remained a trend for sigma to predict performance recovery. Future studies will have to examine these issues more closely. Nonetheless, delta and sigma power appear to influence the degree of inhibitory performance recovery, and each appear to explain distinct portions of the performance variance.

Relationships between the physiology of recovery sleep and the recovery of cognitive function have been understudied, but the current results provide a basis for future research. We found that delta and sigma power changes predicted the degree of inhibitory performance recovery. This relationship was further linked with how delta and sigma were associated with prefrontal functioning. The ability to increase delta power during NREM sleep appears to improve inhibitory performance recovery, whereas increasing sigma power hampers inhibitory performance recovery. One implication is that methods to increase delta power, such as that proposed by Marshall et al. (2004), may improve the speed and degree of inhibitory performance recovery after extended wakefulness.

\section{References}

Aguirre GK, Zarahn E, D’Esposito M (1998) The inferential impact of global signal covariates in functional neuroimaging analyses. Neuroimage 8:302-306.

Bellgrove MA, Hester R, Garavan H (2004) The functional neuroanatomical correlates of response variability: evidence from a response inhibition task. Neuropsychologia 42:1910-1916.

Bjorness TE, Kelly CL, Gao T, Poffenberger V, Greene RW (2009) Control and function of the homeostatic sleep response by adenosine A1 receptors. J Neurosci 29:1267-1276.

Blake H, Gerard RW (1937) Brain potentials during sleep. Am J Physiol 119:692-703. 
Bonnet MH (1985) Recovery of performance during sleep following sleep deprivation in older normal and insomniac adult males. Percept Mot Skills 60:323-334.

Booth JR, Burman DD, Meyer JR, Lei Z, Trommer BL, Davenport ND, Li W, Parrish TB, Gitelman DR, Mesulam MM (2003) Neural development of selective attention and response inhibition. Neuroimage 20:737-751.

Borbély AA, Baumann F, Brandeis D, Strauch I, Lehmann D (1981) Sleep deprivation: effect on sleep stages and EEG power density in man. Electroencephalogr Clin Neurophysiol 51:483-495.

Brett M, Anton JL, Valabregue R, Poline JB (2002) Region of interest analysis using an SPM toolbox [abstract]. In: 8th international conference on functional mapping of the human brain, Vol 16, No 2. Sendai, Japan: Neuroimage.

Cajochen C, Foy R, Dijk DJ (1999) Frontal predominance of a relative increase in sleep delta and theta EEG activity after sleep loss in humans. Sleep Res Online 2:65-69.

Carskadon MA, Dement W (1994) Normal human sleep: an overview. In: Principles and practice of sleep medicine, Ed 2 (Kryger MH, Roth T, Dement W, eds), pp 16-25. Philadelphia: W.B. Saunders.

Chuah YM, Venkatraman V, Dinges DF, Chee MW (2006) The neural basis of interindividual variability in inhibitory efficiency after sleep deprivation. J Neurosci 26:7156-7162.

De Gennaro L, Ferrara M (2003) Sleep spindles: an overview. Sleep Med Rev 7:423-440.

Dijk DJ, Hayes B, Czeisler CA (1993) Dynamics of electroencephalographic sleep spindles and slow wave activity in men: effect of sleep deprivation. Brain Res 626:190-199.

Garavan H, Ross TJ, Stein EA (1999) Right hemispheric dominance of inhibitory control: an event-related functional MRI study. Proc Natl Acad Sci U S A 96:8301-8306.

Gosselin A, De Koninck J, Campbell KB (2005) Total sleep deprivation and novelty processing: implications for frontal lobe functioning. Clin Neurophysiol 116:211-222.

Halassa MM, Florian C, Fellin T, Munoz JR, Lee SY, Abel T, Haydon PG, Frank MG (2009) Astrocytic modulation of sleep homeostasis and cognitive consequences of sleep loss. Neuron 61:213-219.

Harrison Y, Horne JA, Rothwell A (2000) Prefrontal neuropsychological effects of sleep deprivation in young adults-a model for healthy aging? Sleep 23:1067-1073.

Hester RL, Murphy K, Foxe JJ, Foxe DM, Javitt DC, Garavan H (2004) Predicting success: patterns of cortical activation and deactivation prior to response inhibition. J Cogn Neurosci 16:776-785.

Holland PW, Welsch RE (1977) Robust regression using iteratively reweighted least-squares. Commun Stat Theory Methods 6:813-827.

Horn NR, Dolan M, Elliott R, Deakin JF, Woodruff PW (2003) Response inhibition and impulsivity: an fMRI study. Neuropsychologia 41:19591966.

Kales A, Tan TL, Kollar EJ, Naitoh P, Preston TA, Malmstrom EJ (1970) Sleep patterns following 205 hours of sleep deprivation. Psychosom Med 32:189-200.

Kleitman N (1963) Sleep and wakefulness. Chicago: University of Chicago Press.

Konishi S, Nakajima K, Uchida I, Kikyo H, Kameyama M, Miyashita Y (1999) Common inhibitory mechanism in human inferior prefrontal cortex revealed by event-related functional MRI. Brain 122:981-991.
Macey PM, Macey KE, Kumar R, Harper RM (2004) A method for removal of global effects from fMRI time series. Neuroimage 22:360-366.

MacKinnon DP, Warsi G, Dwyer JH (1995) A simulation study of mediated effect measures. Multivariate Behav Res 30:41-62.

Marshall L, Mölle M, Hallschmid M, Born J (2004) Transcranial direct current stimulation during sleep improves declarative memory. J Neurosci 24:9985-9992.

Massimini M, Huber R, Ferrarelli F, Hill S, Tononi G (2004) The sleep slow oscillation as a traveling wave. J Neurosci 24:6862-6870.

Menon V, Adleman NE, White CD, Glover GH, Reiss AL (2001) Errorrelated brain activation during a Go/NoGo response inhibition task. Hum Brain Mapp 12:131-143.

Oldfield RC (1971) The assessment and analysis of handedness: the Edinburgh inventory. Neuropsychologia 9:97-113.

Patrick GTW, Gilbert JA (1896) On the effects of loss of sleep. Psychol Rev 3:468-483.

Rechtschaffen A, Kales A (1968) A manual of standardized terminology, techniques an scoring system of sleep stages in human subjects. Los Angeles: UCLA Brain Information Services.

Rosa RR, Bonnet MH, Warm JS (1983) Recovery of performance during sleep following sleep deprivation. Psychophysiology 20:152-159.

Schulze G (2004) Sleep protects excitatory cortical circuits against oxidative damage. Med Hypotheses 63:203-207.

Steriade M, Domich L, Oakson G, Deschenes M (1987) The deafferented reticular thalamic nucleus generates spindle rhythmicity. J Neurophysiol 57:260-273.

Steriade M, Contreras D, Curró Dossi R, Nuñez A (1993) The slow $(<1 \mathrm{~Hz})$ oscillation in reticular thalamic and thalamocortical neurons: scenario of sleep rhythm generation in interacting thalamic and neocortical networks. J Neurosci 13:3284-3299.

Tailairach J, Tournoux P (1988) Steriotaxic atlas of the human brain. New York: Thieme Medical.

Thomas M, Sing H, Belenky G, Holcomb H, Mayberg H, Dannals R, Wagner H, Thorne D, Popp K, Rowland L, Welsh A, Balwinski S, Redmond D (2000) Neural basis of alertness and cognitive performance impairments during sleepiness. I. Effects of $24 \mathrm{~h}$ of sleep deprivation on waking human regional brain activity. J Sleep Res 9:335-352.

Tononi G, Cirelli C (2003) Sleep and synaptic homeostasis: a hypothesis. Brain Res Bull 62:143-150.

Walker MP, Liston C, Hobson JA, Stickgold R (2002) Cognitive flexibility across the sleep-wake cycle: REM-sleep enhancement of anagram problem solving. Brain Res Cogn Brain Res 14:317-324.

Walker MP, Stickgold R, Alsop D, Gaab N, Schlaug G (2005) Sleepdependent motor memory plasticity in the human brain. Neuroscience 133:911-917.

Watanabe J, Sugiura M, Sato K, Sato Y, Maeda Y, Matsue Y, Fukuda H, Kawashima R (2002) The human prefrontal and parietal association cortices are involved in NO-GO performances: an event-related fMRI study. Neuroimage 17:1207-1216.

Williams HL, Lubin A, Goodnow JJ (1959) Impaired performance with acute sleep loss. Psychol Monogr 73:484.

Wu JC, Gillin JC, Buchsbaum MS, Chen P, Keator DB, Khosla Wu N, Darnall LA, Fallon JH, Bunney WE (2006) Frontal lobe metabolic decreases with sleep deprivation not totally reversed by recovery sleep. Neuropsychopharmacology 31:2783-2792. 